\title{
Electron hopping conduction in highly disordered carbon coils
}

\author{
Hui-Shan Chiu ${ }^{a}$, Ping-I Lin ${ }^{b}$, Hsing-Chen Wu ${ }^{a}$, Wen-Hsing Hsieh ${ }^{c}$, Chii-Dong Chen ${ }^{c, d, *}$, \\ Yit-Tsong Chen ${ }^{a, b, *}$
}

${ }^{a}$ Department of Chemistry, National Taiwan University, No. 1, Sec. 4, Roosevelt Road, Taipei 106, Taiwan

binstitute of Atomic and Molecular Sciences, Academia Sinica, Taipei 106, Taiwan

'Institute of Physics, Academia Sinica, Nankang, Taipei 11529, Taiwan

${ }^{\mathrm{d}}$ Department of Physics, National Cheng Kung University, Tainan 701, Taiwan

\section{A R T I C L E I N F O}

Article history:

Received 8 October 2008

Accepted 1 March 2009

Available online 9 March 2009

\begin{abstract}
A B S T R A C T
Carbon coils of micrometer to nanometer wire diameter were grown bi-directionally by catalyst-assisted chemical vapor deposition. Electron microscope images showed the highly disordered structure of the carbon coils. Chemical compositions of the coils were identified with elemental analysis, atmospheric pressure-laser desorption ionizationFourier-transform ion cyclotron resonance-mass spectroscopy and secondary ion mass spectrometric characterizations, and attenuated total reflection-infrared spectroscopic examination. Micro-Raman scattering spectroscopy and electron energy loss spectroscopy were also used to study the vibrational and electronic properties of the helical structure. The electric transport in a single carbon coil was measured from ambient temperature to $64 \mathrm{mK}$. The temperature-dependent resistance was analyzed with the Efros-Shklovskii variable range hopping model, indicating three-dimensional electron hopping conduction in the disordered helical wires. The analysis also provides a basic understanding of the electron transport with an electron hopping length of $\sim 5 \mathrm{~nm}$ inside the disordered carbon coils.

(c) 2009 Elsevier Ltd. All rights reserved.
\end{abstract}

\section{Introduction}

Carbon is a mysterious element with several allotropic forms, which can be tailored to various kinds of conformations and morphologies in the micrometer to nanometer scales, such as buckyballs [1], tubes [2], onions [3], calabashes [4], foams [5], fibers [6], rods [7], buds [8], etc. Among these micro/ nano-objects, carbon coils have fascinating helical morphology and possess many unique characteristics and novel functionalities, e.g. good chiral conductivity $[9,10]$, large surface area [9], super-elasticity [11]. In practical applications, the helical carbon structures have been used to fabricate semiconducting infrared (IR) detection elements [12], flat panel field emission display [13], and microwave absorber [14].
These carbon coils are also excellent materials for prospective versatile fabrications in micro-devices, micro-magnetic sensors [15], actuators [16], hydrogen adsorption-desorption materials, chiral catalysts, etc.

The catalytic growth mechanisms of carbon coils in chemical vapor deposition (CVD) reactions have long been of great interest in the research of helical/spiral materials. In the past decades, more than a dozen of growth models have been proposed to elucidate the formations of carbon coils with distinctive shapes and morphologies [17]. Several excellent surveys about the preparations, morphology, growth mechanisms, properties, and applications of carbon coils can be found in the reviews by Rodriguez [18], De Jong and Geus [19], and Motojima and Chen [17]. In particular,

\footnotetext{
* Corresponding authors: Address: Department of Chemistry, National Taiwan University, No. 1, Sec. 4, Roosevelt Road, Taipei 106, Taiwan. Fax: +886 223620200 (Y.-T. Chen), +886 227892440 (C.-D. Chen).

E-mail addresses: chiidong@phys.sinica.edu.tw (C.-D. Chen), ytchen@pub.iams.sinica.edu.tw (Y.-T. Chen). 0008-6223/\$ - see front matter @ 2009 Elsevier Ltd. All rights reserved. doi:10.1016/j.carbon.2009.03.002
} 
the most recent review by Motojima and Chen [17] made a thorough survey of a myriad of growth models proposed for the fabrication of carbon coils in metal-catalyst-assisted CVD reactions.

While the catalytic growths and structural characterizations of carbon coils have been extensively investigated since the early studies decades ago [20,21], the measurements of electric transport in the helical carbonaceous structure were relatively few in number. To the best of our knowledge, the electric properties of carbon coils have been studied exclusively by Motojima's group [17]. The electric conductivity of a single piece of carbon micro-coil with the diameter of several micrometers and the length of several millimeters was measured 30-100 S/cm, where the temperature dependence ( $400-10 \mathrm{~K})$ of conductivity indicated both semi-conductive behavior with an activation energy of $4 \mathrm{meV}$ and a variable range hopping (VRH) transport mechanism in the carbon micro-coil [10].

In this paper, we first present the bi-directionally grown carbon coils in metal-catalyst-assisted CVD experiments with acetylene as a reaction precursor. We have taken various electron microscopic images of the carbon coils to study their disordered microstructures. Chemical compositions of the coils were identified by elemental analysis (EA), mass spectrometric (MS) characterizations, and IR spectroscopic examination. Micro-Raman scattering spectroscopic and electron energy loss spectroscopic (EELS) investigations of the carbon coils further reveal the vibrational and electronic properties of the disordered structure. In addition to the structural and spectroscopic studies, we measured the electric transport in a single carbon coil in a temperature range from $280 \mathrm{~K}$ to $64 \mathrm{mK}$. The resistance of the carbon coil at $6 \mathrm{~K}<\mathrm{T}<94 \mathrm{~K}$ can be described by the Efros-Shklovskii VRH conduction (ES-VRHC) model in three-dimensions with an electron hopping length of $\sim 5 \mathrm{~nm}$ inside the highly disordered structure.

\section{Experimental}

\subsection{Catalytic synthesis}

Apparatus used for the catalyst-assisted CVD reaction to bidirectionally grow carbon coils is illustrated in Fig. 1, of which the equipments are similar to those used for the recent syntheses of Si nanowires [22], CdSe nanobelts and nanosheets [23], and $\mathrm{Zn}_{\mathrm{x}} \mathrm{Cd}_{1-\mathrm{x}}$ Se alloy nanowires [24] in our laboratory. In the catalyst-assisted CVD reactions, a piece of $\mathrm{Cu}$ foil (Strem Chemicals, Cu $99.9 \%, 15 \times 5 \times 0.03 \mathrm{~mm}^{3}$ ) and a Ni plate (Nilaco, Ni $99.7 \%, 500 \times 18 \times 3 \mathrm{~mm}^{3}$ ) were placed inside a quartz tube ( $2 \mathrm{~cm}$ inner diameter and $80 \mathrm{~cm}$ long). The system was heated by a temperature-controlled tubular electric furnace (50 cm long). The $\mathrm{Cu}$ foil, lying on the $\mathrm{Ni}$ plate, was placed downstream to the flow of gaseous reacting precursors. Before reaction, the system was evacuated to a few mtorr. Temperature of the system was elevated gradually to the synthesis condition at $\sim 800^{\circ} \mathrm{C}$. At this temperature, the vacuum-annealed surface film of the $\mathrm{Cu}$ foil was recrystallized to $\mathrm{Cu}$ particles of $\sim 100-200 \mathrm{~nm}$ in diameter. A gas mixture of acetylene (Matheson, $\mathrm{C}_{2} \mathrm{H}_{2}$ 99.6\%) and argon
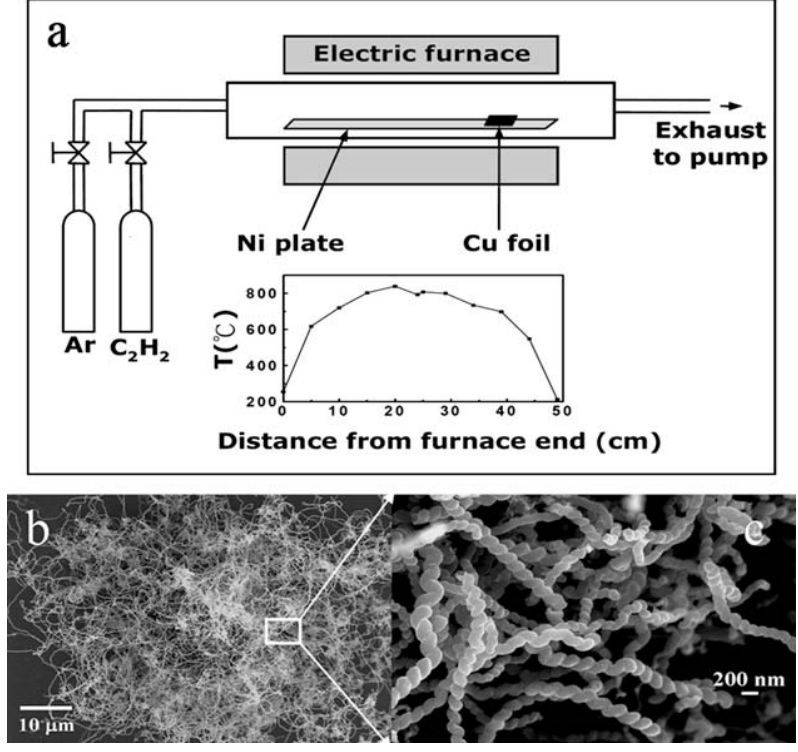

Fig. 1 - Catalytic growth of carbon coils. (a) The experimental setup for the CVD reactions to synthesize carbon coils. A temperature profile inside the electric furnace during the reaction is presented. $(b-c)$ Abundant carbon coils have been synthesized on the copper foil with high product yield. The carbon coils have uniform wire diameter, pitch length, and helix radius.

(Matheson, Ar 99.999\%) with a pressure ratio of 1:1 was then introduced to the quartz tube. The gas flow rate was maintained at 10-15 sccm with a total pressure of 50 Torr throughout the reaction. The optimal deposition temperature for the growth of carbon coils in this study is at $700-750{ }^{\circ} \mathrm{C}$. After the reaction, a large number of carbon coils were produced on the $\mathrm{Cu}$ foil. The high product yield of carbon coils is demonstrated in Fig. $1 \mathrm{~b}$ and $\mathrm{c}$.

\subsection{Electron microscopic and spectroscopic characterizations}

Electron microscopic characterizations of the as-synthesized carbon coils were performed by scanning electron microscopy (SEM), energy dispersive X-ray spectroscopy (EDS) (LEO 1530, Filed Emission Gun SEM + EDS), transmission electron microscopy (TEM), selected-area electron diffraction (SAED), and high-resolution TEM (HRTEM) (JEOL JEM 2010 Analytical TEM at $200 \mathrm{kV}$ and JEOL 4000 EX HRTEM at $400 \mathrm{kV}$ ). Room-temperature micro-Raman spectroscopic experiments were carried out in a confocal microscope (Jobin Yvon, 3143MFO) using an Ar-Kr ion laser (Coherent, Innova 70C) at $514.5 \mathrm{~nm}$ as an excitation light source. The laser beam was focused into a spot of $\sim 4 \mu \mathrm{m}$ in diameter on the sample of carbon coils and the Raman scattering signals, detected in a $180^{\circ}$ backscattering geometry, were sent into a monochromator (Jobin Yvon, Triax 550) equipped with a 1800 grooves/mm grating. The spectral data were finally colleted by a liquid-nitrogen cooled charge-coupled device camera (Jobin Yvon, Spectrum-ONE, $1024 \times 256$ pixels). The EELS spectra of graphite and carbon 
coils were taken in a TEM equipped with EELS (JEOL, JEM2100F) having an electron-beam probe size of $\sim 5 \AA$ in diameter. The spatial and energy resolutions of the EELS are a few $\AA$ and $0.2 \mathrm{eV}$, respectively.

\subsection{Chemical compositions}

EA for the as-synthesized carbon coils was conducted in a commercial analyzer (EA, Heraeus VarioEL-III). Mass analysis for the fragmentary species of carbon coils was performed with an atmosphere pressure-laser desorption ionizationFourier-transform ion cyclotron resonance-mass (AP-LDIFTICR-MS) spectrometer, of which the experimental details can be found in the work by Sabu et al. [25]. Briefly, the carbon coils were dispersed in ethanol solution assisted with sonication. The concentrated solution containing carbon coils, after a centrifugation treatment, was dropped on a graphite target and dried in air. In the experiments, the graphite target was used as a blank background for carbon. A $\mathrm{N}_{2}$ laser (SpectraPhysics, VLS-337ND) at $337 \mathrm{~nm}$ with the maximum pulse energy of $300 \mu \mathrm{J}$ and repetition rate of $20 \mathrm{~Hz}$ was employed to irradiate the sample plate at an incidence angle of $60^{\circ}$ with respect to the surface normal of the target plate. The fragments of carbon coils in atmosphere pressure (AP) after laser desorption and ionization (LDI) were then guided to enter a Fouriertransform ion cyclotron resonance-mass (FTICR-MS) spectrometer equipped with a 7.0-T actively shielded magnet and Apollo electrospray interface (Bruker-Daltonics, Apex III). The FTICR-MS can be used to detect the chemical species of $\mathrm{m} / \mathrm{z}=45-4000$ with a mass accuracy of $\sim 5 \mathrm{ppm}$. The masses of the fragmentary species and their isotopic intensity ratios were analyzed with the Bruker-Daltonics software packages.

\subsection{Device fabrication and characterization}

The lithographic fabrication and electric characterization of carbon coils are similar to those of single-walled carbon nanotube field-effect transistors carried out recently in our laboratory [26,27]. Briefly, individual carbon coils were dispersed and placed on thermally oxidized silicon chips with predefined micrometer-sized Au contact pads made by standard photography techniques. The positions of carbon coils were accurately determined by SEM inspections. Subsequently, $100 \mathrm{~nm}$ thick and $150 \mathrm{~nm}$ wide $\mathrm{Cr}$ contact electrodes were designed and made on the top of the carbon coils by electron-beam lithography. $\mathrm{Cr}$ leads have proven capability to provide good electric contact with carbon [28]. Each carbon coil was connected to several leads, allowing four-probe measurements to be made on successive segments between the two central probes. The transport characteristics were measured using a 2-stage close-cycle RDK-205D (Sumitomo Heavy Industries) and a $\mathrm{He}^{3} / \mathrm{He}^{4}$ dilution refrigerator (Air Liquide). To take the full advantages of common-mode noise rejection, the measurement setup, including biasing circuitry and the differential current/voltage amplifiers, is symmetric with respect to the ground. The electrical signal outputs from the amplifiers were taken by Keithley bench-type digital multimeters controlled by LabView programs.

\section{Results and discussion}

\subsection{Electron microscopic images}

Fig. 2a shows an SEM image of the bi-directionally grown carbon coils, of which the pair has right (R)- and left (L)handed chiralities on each side. A catalytic $\mathrm{Cu}$ particle embedded in the joint as an enantiomeric center, interconnecting the two helixes of different chiralities, will be seen more clearly later in a TEM image. The labeled numbers are provided a guide for the eye to count the helical pitches with a symmetric manner on both sides. Fig. $2 b$ displays another example of the as-synthesized carbon coils with uniform wire diameter, pitch length, and helix radius. (More examples of bi-directionally grown carbon coils are presented in Supporting Information S1.)

Further electron microscopic characterizations for the microstructure of carbon coils are shown in Fig. 3. The TEM image of a pair of carbon coils is presented in Fig. 3a. The chemical composition of the catalytic $\mathrm{Cu}$ particle is analyzed by EDS (Fig. 3b). SAED pattern of the Cu particle (Fig. 3c) further indicates its face-centered cubic (fcc) crystalline structure. The carbon constituent in the coils is identified from an EDS analysis (Fig. 3d). SAED pattern (Fig. 3e) and HRTEM images (Fig. $3 f$ and $g$ ) further indicate the highly disordered structure inside the coils as studied previously [29-33].
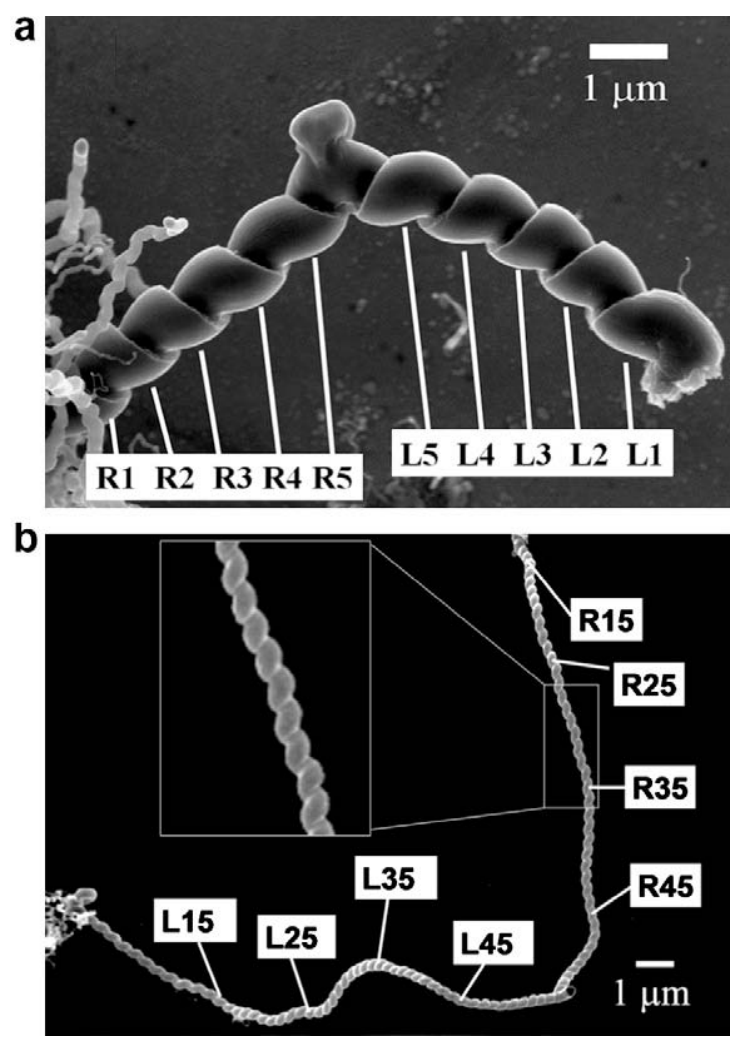

Fig. 2 - SEM images of the as-synthesized carbon coils depict right (R)- and left (L)-handed chiralities on each side with (a) 5 and (b) 53 pitches. The labeled numbers are provided a guide for the eye to count the helical pitches. 


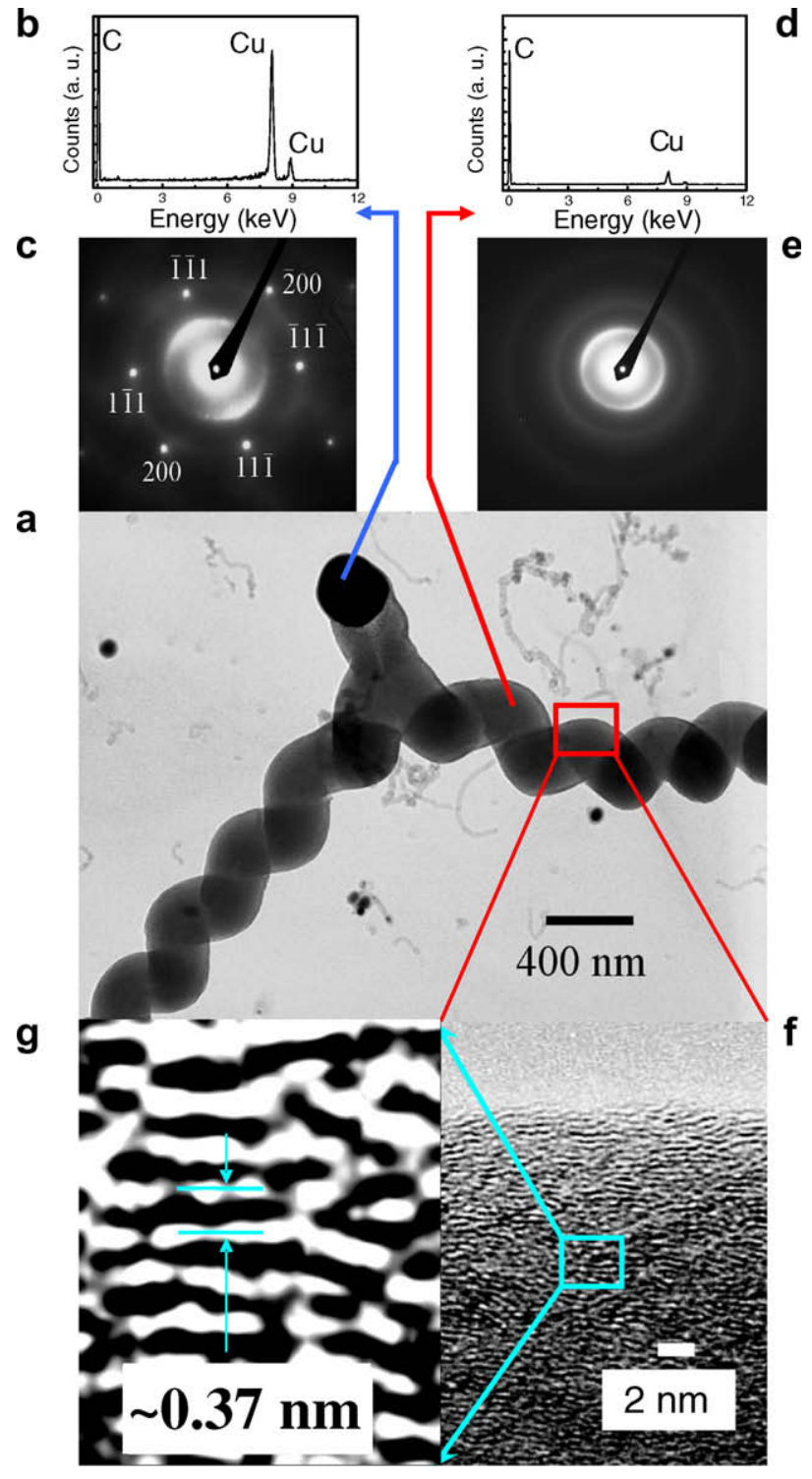

Fig. 3 - Electron microscopic characterizations of carbon coils. (a) TEM image of a pair of carbon coils shows the dimensions of $\sim 300 \mathrm{~nm}$ wire diameter, $\sim 60 \mathrm{~nm}$ helix radius, and $\sim 40 \mathrm{~nm}$ pitch length. A catalyst-particle of $\sim 300 \mathrm{~nm}$ diameter (black ball) is located at the enantiomeric center. (b) EDS analysis identifies the catalytic $\mathrm{Cu}$ particle. The carbon signal in the EDS spectrum is attributed to a carbonaceous shell encapsulating the $\mathrm{Cu}$ particle. (c) An fcc crystalline structure of the $\mathrm{Cu}$ particle is determined from the SAED pattern. (d) An EDS analysis was made for the carbon coils. The tiny $\mathrm{Cu}$ signal in the EDS spectrum comes from the $\mathrm{Cu}$ grid used for the TEM measurement. (e-g) Both SAED pattern and HRTEM image indicate a highly disordered structure inside the carbon coils.

\subsection{Chemical compositions}

The chemical compositions of the carbon coils were determined with EA and mass analysis using AP-LDI-FTICR-MS spectrometry. From the EA of the coils, the compositions include carbon and hydrogen with the molar ratio of $\mathrm{C} / \mathrm{H} \sim$
3/1. The hydrocarbon compositions in carbon coils were also reported recently by Qin et al. [34,35]. In Fig. 4a, we further display the hydrocarbon fragments of carbon coils obtained from the AP-LDI-FTICR-MS analysis at $m / z=200-950$. To correctly assign the fragmentary species with very high accuracy, we set a 10 ppm error window as an acceptable uncertainty range in the mass analysis. For instance, the observed signal at $m / z=831.586686$ (magnified in Fig. 4b) has been identified as $\mathrm{C}_{63} \mathrm{H}_{75}$ with a mass error of $0.2 \mathrm{ppm}$, well within the 10 ppm window. Alternatively, should $\mathrm{C}_{61} \mathrm{H}_{71} \mathrm{~N}_{2}$ be assigned to this peak of $\mathrm{m} / \mathrm{z}=831.586686$, a mass error of $-29.9 \mathrm{ppm}$ would far exceed the acceptable $10 \mathrm{ppm}$ error window, although the masses of $\mathrm{C}_{63} \mathrm{H}_{75}$ and $\mathrm{C}_{61} \mathrm{H}_{71} \mathrm{~N}_{2}$ differ only by those of $\mathrm{C}_{2} \mathrm{H}_{4}$ and $\mathrm{N}_{2}$. The peaks at $\mathrm{m} / \mathrm{z}=832.586275$ and 833.576190 are attributed to the isotopes of carbon $\left(\mathrm{C}^{12}\right.$ and $\left.\mathrm{C}^{13}\right)$ and hydrogen $\left(\mathrm{H}^{1}\right.$ and $\left.\mathrm{H}^{2}\right)$ in $\mathrm{C}_{63} \mathrm{H}_{75}$. The intensity ratios among the isotopic species, due to their natural abundances, can further be used to confirm the correct assignment. In Fig. 4b, the observed intensity ratios of $100: 49.1: 20.0 \%$ for the peaks of $\mathrm{C}_{63} \mathrm{H}_{75}$ at $\mathrm{m} / \mathrm{z}=831.586686,832.586275$, and 833.576190 are in satisfactory agreement with the theoretical calculations of 100:69.2:23.6\% (Fig. 4c). More examples for the fragments of carbon coils, like $\mathrm{C}_{61} \mathrm{H}_{71}, \mathrm{C}_{54} \mathrm{H}_{198}$, and $\mathrm{C}_{52} \mathrm{H}_{194}$, analyzed in the AP-LDI-FTICR-MS measurements are presented in Supporting Information S2.1.

In addition to the EA and AP-LDI-FTICR-MS analysis, we have also carried out additional mass and spectroscopic examinations for the carbon coils by secondary ion mass spectrometry (SIMS) and attenuated total reflection (ATR)-IR spectroscopy, respectively, also indicating the hydrocarbon compositions in the helical structure. The obtained SIMS and ATR-IR data of carbon coils are collected in Supporting Information S2.2 and S2.3.

\subsection{Spectroscopic characterizations}

Fig. 5 shows the micro-Raman scattering spectrum of carbon coils at $1100-1800 \mathrm{~cm}^{-1}$ obtained by confocal microscopy with an optical excitation at $514.5 \mathrm{~nm}$. The two characteristic Raman signals include the $D$ band at $1356.9 \mathrm{~cm}^{-1}$ and the $G$ band at $1593.1 \mathrm{~cm}^{-1}$ [36-39]. The $\mathrm{D}$ band corresponds to a radial breathing mode of $\mathrm{A}_{1 \mathrm{~g}}$ symmetry involving phonons near the $\mathrm{K}$ zone boundary of which the intensity is strictly related to the presence of six-fold aromatic rings. This breathing mode is forbidden in perfect graphite and only becomes active in disordered structures. In contrast, the $\mathrm{G}$ band, corresponding to the zone-center phonons of $E_{2 g}$ symmetry, originates from the in-plane bond stretching of pairs of graphitic carbon atoms. This stretching mode occurs at all $\mathrm{sp}^{2}$ sites, not only in six-fold aromatic rings, but also in those of other orders.

The observed Raman scattering signals in Fig. 5 have been fitted with a Lorentzian lineshape for the $\mathrm{D}$ band and an asymmetric Breit-Wigner-Fano (BWF) lineshape [36-38] for the $\mathrm{G}$ band. The broad linewidth $\Gamma=213.2 \mathrm{~cm}^{-1}$ (the full width at half maximum, FWHM) of the D band indicates the structural heterogeneity and disorder inside the carbon coils. The BWF lineshape is given by $I(\omega)=\frac{I_{0}\left[1+2\left(\omega-\omega_{0}\right) / O \Gamma\right]^{2}}{1+\left[2\left(\omega-\omega_{0}\right) / \Gamma\right]^{2}}$, where $I_{0}$ is the band intensity, $\omega_{0}=1593.1 \mathrm{~cm}^{-1}$ is the phonon band frequency, $\Gamma=100 \mathrm{~cm}^{-1}$ is the FWHM of the unweighted Lorentzian (when $Q \rightarrow \infty$ ), and $Q^{-1}=-0.143$ is the BWF cou- 

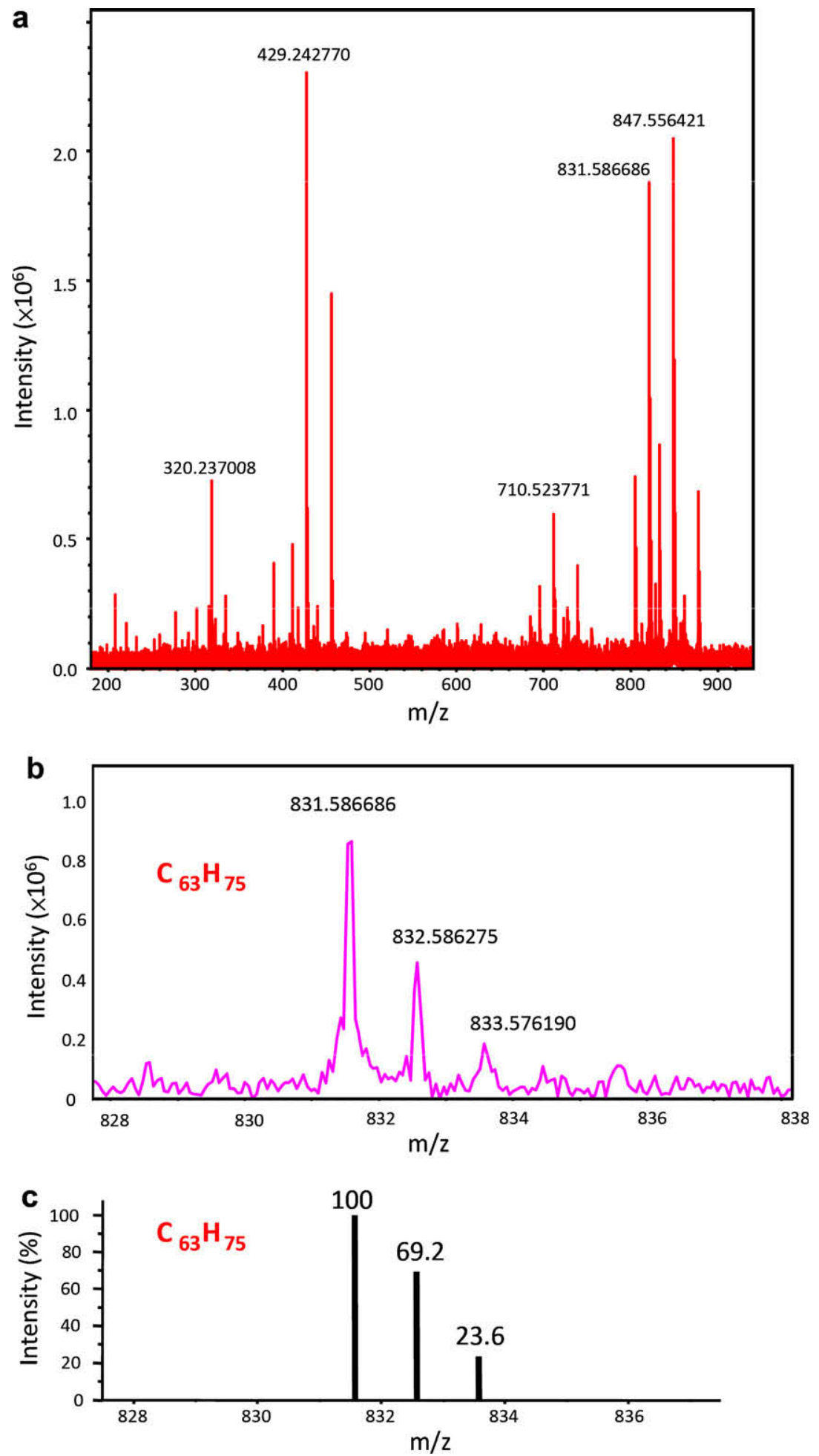

Fig. 4 - (a) Observed AP-LDI-FTICR-MS spectrum of carbon coils at $\mathrm{m} / \mathrm{z}=200-950$. (b) The signal at $\mathrm{m} / \mathrm{z}=831.586686$ is identified as $\mathrm{C}_{63} \mathrm{H}_{75}$ with a mass error of $0.2 \mathrm{ppm}$. Peaks at $\mathrm{m} / \mathrm{z}=832.586275$ and 833.576190 are attributed to the isotopes of carbon $\left(\mathrm{C}^{12}\right.$ and $\left.\mathrm{C}^{13}\right)$ and hydrogen $\left(\mathrm{H}^{1}\right.$ and $\left.\mathrm{H}^{2}\right)$ in $\mathrm{C}_{63} \mathrm{H}_{75}$. (c) The calculated intensity ratios of 100:69.2:23.6\% for the isotopic $\mathrm{C}_{63} \mathrm{H}_{75}$ are in satisfactory agreement with the observed 100:49.1:20.0\% in (b).

pling coefficient relating to the interaction between the discrete $\mathrm{E}_{2 \mathrm{~g}}$ mode and the Raman-active continuum and also indicating the degree of disorder. The microcrystallite size of a graphitic system in the disordered carbon structure can be estimated from the intensity ratio of the $D$ and $G$ bands $\left(I_{D} / I_{G}\right)$ by the empirical formula of $L_{a}=C\left(I_{D} / I_{G}\right)^{-1}[36,39,40]$, where $L_{a}$ is the crystallite size and $C=4.4 \mathrm{~nm}$ at $514.5 \mathrm{~nm}$ excitation. From the intensity ratio of $I_{D} / I_{G}=1.6$ in Fig. 5, a 


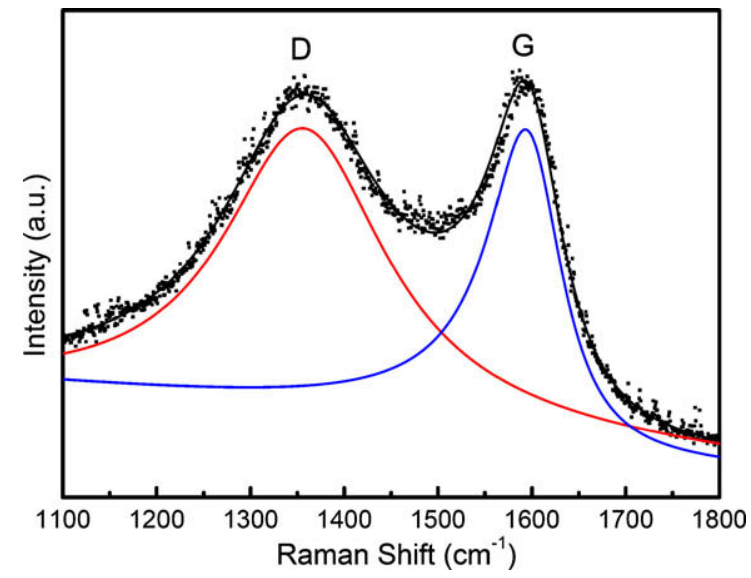

Fig. 5 - Micro-Raman scattering spectrum of a single pair of carbon coils. The red and blue curves represent the $D$ $\left(1356.9 \mathrm{~cm}^{-1}\right)$ and $\mathrm{G}\left(1593.1 \mathrm{~cm}^{-1}\right)$ bands deconvoluted with Lorentzian $\left(\Gamma=213.2 \mathrm{~cm}^{-1}\right)$ and BWF $\left(Q^{-1}=-0.143\right.$ and $\Gamma=100 \mathrm{~cm}^{-1}$ ) lineshapes, respectively. The dots in the graph indicate raw spectral data with a black solid line illustrating the fitted curve. (For interpretation of the references to colour in this figure legend, the reader is referred to the web version of this article.)

rough estimate of the crystallite size of about $3 \mathrm{~nm}$ was obtained.

Fig. 6 shows the EELS spectra of graphite and carbon coils. The EELS features of graphite are fitted with two Gaussian bands of the band centers at $285 \mathrm{eV}\left(1 \mathrm{~s}-\pi^{*}\right.$, red line) and $292 \mathrm{eV}\left(1 \mathrm{~s}-\sigma^{*}\right.$, green line) [41,42]. These $1 \mathrm{~s}-\pi^{*}$ and $1 \mathrm{~s}-\sigma^{*}$ bands serve as a reference to analyze the EELS spectrum of carbon coils, where the deconvoluted bands were tuned to accommodate the rising edges of the $1 s-\pi^{*}$ and $1 s-\sigma^{*}$ transitions. In

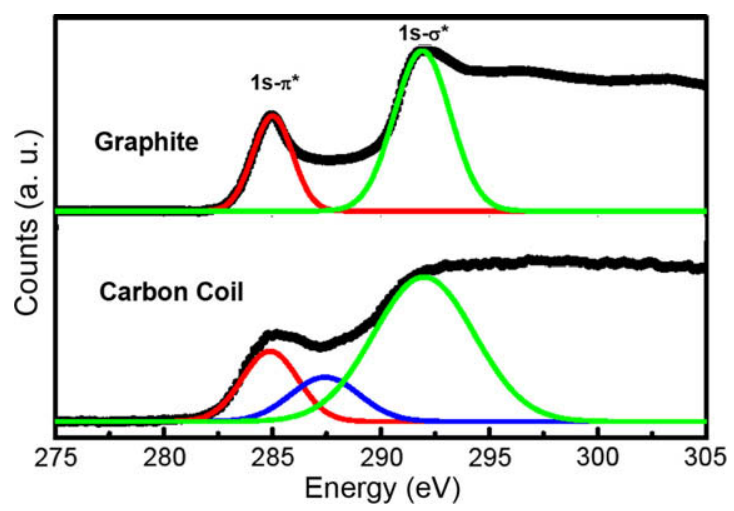

Fig. 6 - EELS spectra of graphite and carbon coils. The obtained EELS spectra were deconvoluted with Gaussian lineshapes, where the red bands at $285 \mathrm{eV}$ represent the $1 \mathrm{~s}-$ $\pi^{*}$ transition, the green bands at $292 \mathrm{eV}$ correspond to the $1 \mathrm{~s}-\sigma^{*}$ transition, and the $\mathbf{C}-\mathbf{H}$ bonds in the disordered graphitic systems of carbon coils are responsible for the blue band at $287.4 \mathrm{eV}$. (For interpretation of the references to colour in this figure legend, the reader is referred to the web version of this article.)
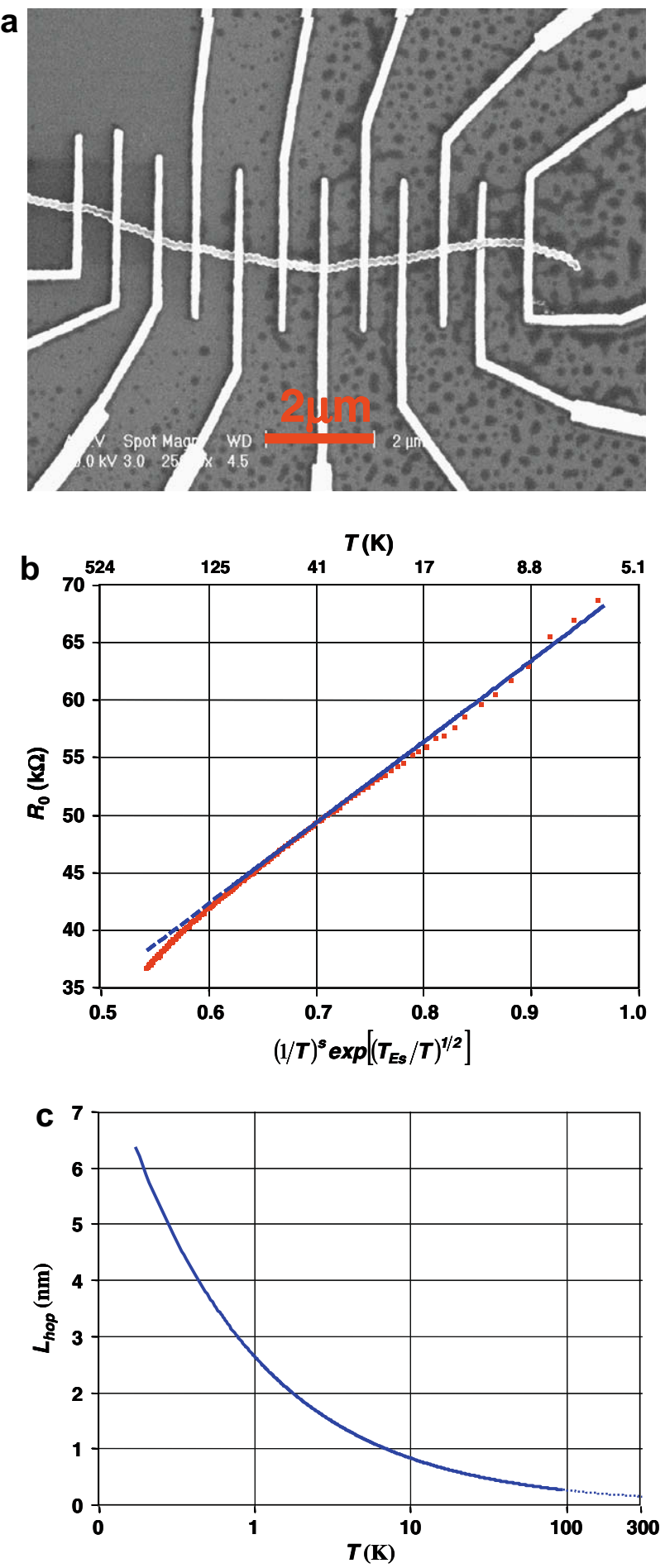

Fig. 7 - Electric transport measurement of carbon coils. (a) An SEM image of the measured carbon coil of $\sim 150 \mathrm{~nm}$ wire diameter. The scale bar is $2 \mu \mathrm{m}$. (b) The $R_{0}(T)$ dependence with a fitting to ES-VRHC behavior at temperatures below $94 \mathrm{~K}$. Dashed curve shows an extension to $300 \mathrm{~K}$. The exponent $s$ and ES-temperature $T_{E S}$ are 0.1136 and $0.1744 \mathrm{~K}$, respectively. The corresponding actual temperatures are labeled at the top for reference. (c) The mean hopping distance as a function of temperature, in which the dotted curve shows an extrapolation using the theory of VRHC. 
addition to the $1 \mathrm{~s}-\pi^{*}$ and $1 \mathrm{~s}-\sigma^{*}$ bands in the EELS spectrum of carbon coils, there remains a weak feature in between and has been deconvoluted to center at $287.4 \mathrm{eV}$ (blue line). This weak band is attributed to the contaminant $\mathrm{C}-\mathrm{H}$ bonds in disordered graphitic systems $[41,43]$. The hydrogens inside the carbon coils might result from those hydrogen atoms acting as a terminator to the dangling carbons on the edges of disordered graphite $[44,45]$.

\subsection{Electric conduction}

Electric transport measurements for a single carbon coil have been carried out in a wide temperature range from $280 \mathrm{~K}$ to $64 \mathrm{mK}$. Fig. 7a and $\mathrm{b}$ shows an SEM image of the measured carbon coil of $\sim 150 \mathrm{~nm}$ wire diameter and its zero-bias resistance $\left(R_{\circ}\right)$ at temperatures between 280 and $6 \mathrm{~K}$. The resistance increases no more than 2-times as the temperature decrease by almost 2-orders of magnitude. This small increase in the resistance suggests that the behavior may be described by the hopping conductivity on the insulating side but quite close to the critical regime of the metal-insulator transition [46]. Indeed, it is found that the resistance at low temperatures can be very well described by the ES-VRHC model [47]. In this model, the Coulomb interaction between hopping sites was considered, and the resistance as a function of temperature reads

$R_{0}(T)=R_{a}(1 / T)^{s} \exp \left[\left(T_{E S} / T\right)^{1 / 2}\right]$
Using the exponent $s$ as a free parameter, one can find a set of best prefactor $R_{a}$ and ES-characteristic temperature $\mathrm{T}_{\mathrm{ES}}$ in an appropriate temperature range for a minimum deviation from the measured $R_{0}(T)$ curve. It is found that a minimum deviation is achieved for $s=0.11$ in the temperature range between 6 and $94 \mathrm{~K}$, and the corresponding $R_{a}$ and $T_{E S}$ values are estimated to be $70.5 \mathrm{k} \Omega$ and $0.17 \mathrm{~K}$, respectively. The latter reflects the strength of the Coulomb interaction and is related to the localization length $\xi$ and static dielectric response $\varepsilon^{\prime}$ as

$k_{\mathrm{B}} \mathrm{T}_{\mathrm{ES}} \approx \beta e^{2} / \xi \varepsilon^{\prime}$

$\beta$ is a constant of approximately 2.8. The dielectric response

$\varepsilon^{\prime}=\varepsilon_{0} \varepsilon_{r}=\varepsilon_{\text {host }}+4 \pi e^{2} \mathrm{~N}\left(E_{\mathrm{F}}\right) \xi^{2}$

is composed of the normal host lattice dielectric response $\varepsilon_{\text {host }}$ and an anomalous contribution related to the density of states at the Fermi-level $N\left(E_{F}\right)$ and the localization length $\xi . \varepsilon_{\mathrm{o}}=8.85 \times 10^{-12} \mathrm{~F} / \mathrm{m}$ is the permittivity constant. Near the metal-insulator transition, the anomalous contribution is expected to dominate the dielectric response, and $\mathrm{T}_{\mathrm{ES}}$ can be rewritten as $k_{\mathrm{B}} \mathrm{T}_{\mathrm{ES}} \approx(2.8 / 4 \pi) N\left(E_{\mathrm{F}}\right) \xi^{3}$. Note that, apart from a numeric factor the ES-characteristic temperature can be understood as the mean energy-level-spacing $N\left(E_{\mathrm{F}}\right) \xi^{3}$ of a localized system. With the knowledge of $T_{E S}$ and a $N\left(E_{F}\right)$ value of $4.4 \times 10^{20} / \mathrm{eV} / \mathrm{cm}^{3}$ [48], we estimated the localization length $\xi$ to be $32 \mathrm{~nm}$. Accordingly, a $\varepsilon_{\mathrm{r}}$ value of approximately $10^{6}$ is

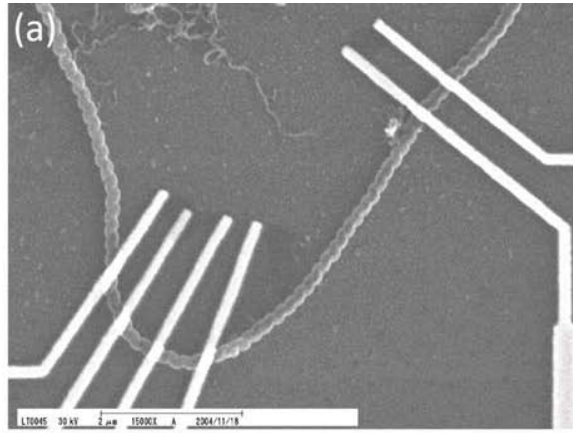

(c)

(d)
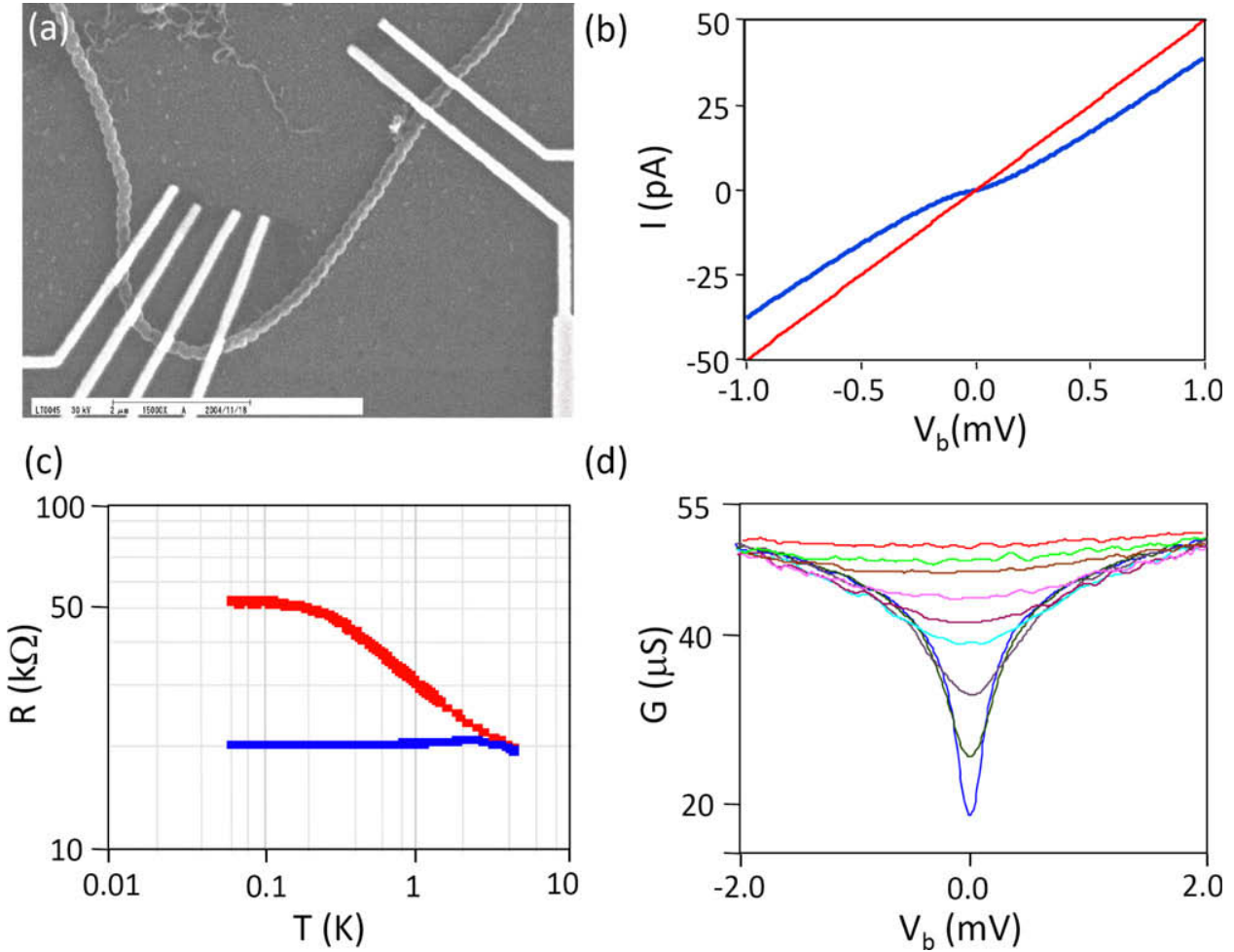

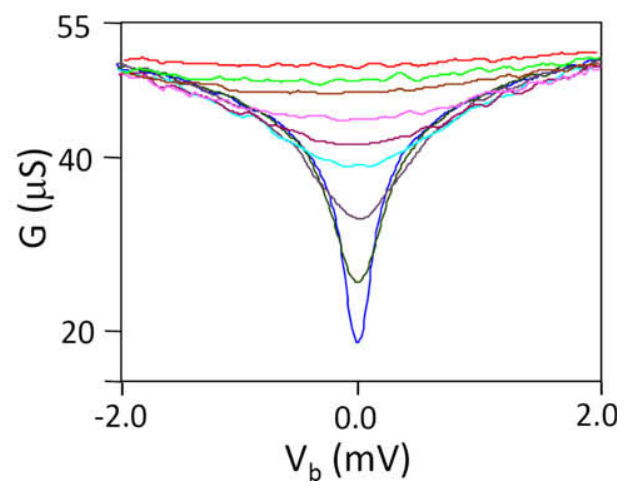

Fig. $8-I-V_{b}$ characteristics of a single carbon coil. (a) The SEM image of a carbon coil device for the electric transport measurements. (b) The red and blue curves are for $\mathrm{T}=4 \mathrm{~K}$ and $64 \mathrm{mK}$, respectively. (c) The asymptotic (blue) and zero-bias (red) resistances are presented as a function of temperature at $T \leqslant 4 \mathrm{~K}$, which is the onset temperature for nonlinear $I-V_{b}$ characteristics. (d) The curves show the dynamic conductance of $d I / d V_{b}$ versus $V_{b}$ at various temperatures, from top 4.0 (red), 3.5, 3.0, 2.5, 2.0, 1.5, 1.0, 0.5, $0.064 \mathrm{~K}$ (blue). (For interpretation of the references to colour in this figure legend, the reader is referred to the web version of this article.) 
implied, which is much great than $\varepsilon_{\text {host }} / \varepsilon_{\mathrm{o}}$ and our assumption that the host dielectric response can be neglected is justified. A small value of characteristic temperature, $\mathrm{T}_{\mathrm{ES}} \approx 0.17 \mathrm{~K}$, is consistent with the theory for the ES-VRHC. In the critical regime, the value of exponent $s$ may be substantially altered and a comparison with the theory may not be practical. Since the localization length is much smaller than the helix diameter ( $\sim 150 \mathrm{~nm}$, see the SEM image in Fig. 7a), the electric transport in the carbon coils is governed by three-dimensional hopping conduction inside the disordered helical structure. For the VRHC theory to be valid, the localization length should be considerably greater than the nearest-neighbor site separation. This is fulfilled because the localization length $(\xi \approx 32 \mathrm{~nm})$ is much greater than the crystallite size of $3 \mathrm{~nm}$ estimated from the Raman scattering analysis. Note that the localization length $\xi$ is expected to diverge as the metalinsulator transition is approached from the insulating side [49]. Further, as shown in Fig. 7c, the hopping length $L_{h o p}(T)$ increases with decreasing temperature as $L_{\text {hop }}(T)=(1 / 4) \xi\left(T_{E S} / T\right)^{1 / 2}$. In view of that, the transition may be imperfect at very low temperatures due to finite diameter of the carbon helix wires. The hopping conduction in our carbon coils is in line with that reported in highly disordered activated carbon-fibers [39] where the resistivity follows $\exp \left[\left(T_{0} / T\right)^{1 / 2}\right]$ dependence and is attributed to charge-energy-limited tunnelling conduction rather than 1D-VRHC because of the unphysical fitting parameters.

Fig. $8 \mathrm{a}$ and $\mathrm{b}$ shows the SEM image of another carbon coil device and its current-voltage $\left(I-V_{b}\right)$ curves. The $I-V_{b}$ characteristics at room temperature for all measured carbon coils are linear within tens of millivolts, and the resistance values are in the range of a few $\mathrm{k} \Omega$. Upon cooling, the resistance increases but the $I-V_{b}$ characteristics remains linear at temperature above $4 \mathrm{~K}$. Below $4 \mathrm{~K}$ a concave at the low bias voltage regime starts to develop, leading to an increase of zero-bias resistance $R_{0}$. Fig. 8b shows a comparison of the $I-V_{b}$ characteristics at $4 \mathrm{~K}$ and $64 \mathrm{mK}$ : the lower the temperatures, the more prominent suppression of the current at low bias voltages. While the asymptotic resistance of the carbon coil increases from 19.9 to $20.4 \mathrm{k} \Omega$ (see Fig. 8c), the $R_{\circ}$ shows a deviation from the asymptotic value at $4 \mathrm{~K}$ (i.e. the onset of nonlinear $I-V_{b}$ characteristic) and increases to $53 \mathrm{k} \Omega$ at $64 \mathrm{mK}$. The concave characteristics are best represented by a dip in the differential conductance $G_{d} \equiv d I / d V_{b}$ when plotted as a function of $V_{b}$, as displayed in Fig. 8d for elevated temperatures. The concave characteristics at the low bias region may be attributed to Coulomb interaction between hopping sites as suggested by the ES-VRHC addressed above. Since the devices are in the critical regime of metal-insulator transition, the nonlinear $I-V_{b}$ characteristics cannot be ascribed to strong localization such as Coulomb blockade of electron tunnelling. This is in agreement with the fact that the device showed no gate-voltage dependence which is another hallmark of the Coulomb blockade phenomena. The high conductivity for the carbon coils $(\sim 2.5 \mathrm{mS} / \mu \mathrm{m})$ suggests a high electron concentration and the electron-electron interaction in the coils should be relative weak. In the ES-VRHC theory, a power-law suppression of density of states at the Fermi-level is predicted [47], which may be responsible for the observed concave $I-V_{b}$ characteristics. However, no con- vincing power-law fitting was obtained due probably to the finite wire diameter compared to the diverging localization length at low temperatures.

\section{Conclusion}

Carbon coils have been synthesized bi-directionally from the pyrolysis of acetylene in the catalyst-assisted CVD reaction. The disordered structure of the carbon coils has been investigated by electron microscopy. Furthermore, EA, AP-LDIFTICR-MS, SIMS, and ATR-IR have been applied to analyze the hydrocarbon compositions of the carbon coils. The vibrational and electronic properties of the carbon coils have also been characterized by micro-Raman scattering and EELS spectroscopy. Temperature-dependent resistance for a single carbon coil has been analyzed with the ES-VRHC model, elucidating a three-dimensional electron hopping conduction inside the disordered helical structure. The model analysis elicits a basic understanding of the electric transport with an electron hopping length of $\sim 5 \mathrm{~nm}$ inside the disordered carbon coils, comparable to the crystallite size estimated empirically from the micro-Raman scattering data.

\section{Acknowledgments}

We gratefully acknowledge financial support from the National Science Council of Taiwan, ROC (Grant Nos. NSC 942627-M-002-003 and NSC 95-2112-M-001-062-MY3). Also, we thank Dr. Chau-Chung Han for performing the AP-LDIFTICR-MS experiment and Dr. Shu-Hua Chien for the ATR-IR measurement. Technical support from NanoCore, the Core Facilities for Nanoscience and Nanotechnology at Academia Sinica, is acknowledged.

\section{Appendix A. Supplementary data}

Supplementary data associated with this article can be found, in the online version, at doi:10.1016/j.carbon.2009.03.002.

\section{R E F E R E N C E S}

[1] Kroto HW, Heath JR, Obrien SC, Curl RF, Smalley RE. C-60 Buckminsterfullerene. Nature 1985;318(6042):162-3.

[2] Iijima S. Helical microtubules of graphitic carbon. Nature 1991;354(6348):56-8.

[3] Kroto HW. Carbon allotropes - carbon onions introduce new flavor to fullerene studies. Nature 1992;359(6397):670-1.

[4] Wang ZL, Yin JS. Graphitic hollow carbon calabashes. Chem Phys Lett 1998;289(1-2):189-92.

[5] Rode AV, Hyde ST, Gamaly EG, Elliman RG, McKenzie DR, Bulcock S. Structural analysis of a carbon foam formed by high pulse-rate laser ablation. Appl Phys A-Mater 1999;69:S755-8.

[6] Vieira R, Ledoux MJ, Pham-Huu C. Synthesis and characterisation of carbon nanofibres with macroscopic shaping formed by catalytic decomposition of C2H6/H-2 over nickel catalyst. Appl Catal A-Gen 2004;274(1-2):1-8.

[7] Dubrovinskaia N, Dubrovinsky L, Crichton W, Langenhorst F, Richter A. Aggregated diamond nanorods, the densest and 
least compressible form of carbon. Appl Phys Lett 2005;87(8):083106-1-3.

[8] Nasibulin AG, Anisimov AS, Pikhitsa PV, Jiang H, Brown DP, Choi $\mathrm{M}$, et al. Investigations of nanobud formation. Chem Phys Lett 2007;446(1-3):109-14.

[9] Chen XQ, Motojima S, Iwanaga H. Carbon coatings on carbon micro-coils by pyrolysis of methane and their properties. Carbon 1999;37(11):1825-31.

[10] Kaneto K, Tsuruta M, Motojima S. Electric properties of carbon micro coils. Synthetic Met 1999;103:2578-9.

[11] Chen X, Motojima S, Iwanga H. Vapor phase preparation of super-elastic carbon micro-coils. J Cryst Growth 2002;237:1931-6.

[12] Lakeman CDE, Pan G, Muto N, Miyayama M, Yanagida H, Payne DA. Coiled carbon-fibers from PAN precursors. Mater Lett 1992;13(6):330-5.

[13] Pan LJ, Hayashida T, Zhang M, Nakayama Y. Field emission properties of carbon tubule nanocoils. Jpn J Appl Phys Part 2 2001;40(3B):L235-7.

[14] Motojima S, Hoshiya S, Hishikawa Y. Electromagnetic wave absorption properties of carbon microcoils/PMMA composite beads in W bands. Carbon 2003;41(13):2658-60.

[15] Fujii M, Matsui M, Motojima S, Hishikawa Y. Magnetoresistance in carbon micro-coils annealed at various temperatures. J Cryst Growth 2002;237:1937-41.

[16] Kato Y, Adachi N, Okuda T, Yoshida T, Motojima S, Tsuda T. Evaluation of induced electromotive force of a carbon micro coil. Jpn J Appl Phys Part 1 2003;42(8):5035-7.

[17] Motojima S, Chen X. Nanohelical/spiral materials. In: Nalwa HS, editor. Encyclopedia of nanoscience and nanotechnology. American Scientific Publishers: Valencia, California, USA; 2004. p. 775-94.

[18] Rodriguez NM. A review of catalytically grown carbon nanofibers. J Mater Res 1993;8(12):3233-50.

[19] De Jong KP, Geus JW. Carbon nanofibers: catalytic synthesis and applications. Catal Rev 2000;42(4):481-510.

[20] Davis WR, Slawson RJ, Rigby GR. An unusual form of carbon. Nature 1953;171(4356):756.

[21] Baker RTK, Harris PS, Terry S. Unique form of filamentous carbon. Nature 1975;253(5486):37-9.

[22] Yang YH, Wu SJ, Chiu HS, Lin PI, Chen YT. Catalytic growth of silicon nanowires assisted by laser ablation. J Phys Chem B 2004;108(3):846-52.

[23] Venugopal R, Lin PI, Liu CC, Chen YT. Surface-enhanced Raman scattering and polarized photoluminescence from catalytically grown CdSe nanobelts and sheets. J Am Chem Soc 2005;127(32):11262-8.

[24] Venugopal R, Lin PI, Chen YT. Photoluminescence and Raman scattering from catalytically grown $\mathrm{Zn}_{\mathrm{x}} \mathrm{Cd}_{1-\mathrm{x}} \mathrm{Se}$ alloy nanowires. J Phys Chem B 2006;110(24):11691-6.

[25] Sabu S, Yang FC, Wang YS, Chen WH, Chou MI, Chang HC, et al. Peptide analysis: solid phase extraction-elution on diamond combined with atmospheric pressure matrixassisted laser desorption/ionization-Fourier transform ion cyclotron resonance mass spectrometry. Anal Biochem 2007;367(2):190-200.

[26] Wang CW, Pan CY, Wu HC, Shih PY, Tsai CC, Liao KT, et al. In situ detection of chromogranin A released from living neurons with a single-walled carbon-nanotube field-effect transistor. Small 2007;3:1350-5.

[27] Tsai CC, Yang CC, Shih PY, Wu CS, Chen CD, Pan CY, et al. Exocytosis of a single bovine adrenal chromaffin cell: the electrical and morphological studies. J Phys Chem B 2008;112:9165-73.

[28] Hsiou YF, Yang YJ, Stobinski L, Kuo W, Chen CD. Controlled placement and electrical contact properties of individual multiwalled carbon nanotubes on patterned silicon chips. Appl Phys Lett 2004;84(6):984-6.
[29] Zhang M, Nakayama Y, Pan L. Synthesis of carbon tubule nanocoils in high yield using iron-coated indium tin oxide as catalyst. Jpn J Appl Phys 2000;39(12A):L1242-4.

[30] Jiao J, Einarsson E, Tuggle DW, Love L, Prado J, Coia GM. Highyield synthesis of carbon coils on tungsten substrates and their behavior in the presence of an electric field. J Mater Res 2003;18(11):2580-7.

[31] Zhang GY, Jiang X, Wang EG. Self-assembly of carbon nanohelices: characteristics and field electron emission properties. Appl Phys Lett 2004;84(14):2646-8.

[32] Chen XQ, Yang SM, Motojima S, Ichihara M. Morphology and microstructure of twisting nano-ribbons prepared using sputter-coated Fe-base alloy catalysts on glass substrates. Mater Lett 2005;59(7):854-8.

[33] Liu Q, Cui ZM, Ma Z, Bian SW, Song WG. Carbon materials with unusual morphologies and their formation mechanism. J Phys Chem C 2007;111(33):12420-4.

[34] Qin Y, Zhang ZK, Cui ZL. Helical carbon nanotibers prepared by pyrolysis of acetylene with a catalyst derived from the decomposition of copper tartrate. Carbon 2003;41(15):3072-4.

[35] Qin Y, Yu LY, Wang Y, Li GC, Cui ZL. Amorphous helical carbon nanofibers synthesized at low temperature and their elasticity and processablity. Solid State Commun 2006;138(1):5-8.

[36] Ferrari AC, Robertson J. Interpretation of Raman spectra of disordered and amorphous carbon. Phys Rev B 2000;61(20):14095-107.

[37] McCulloch DG, Prawer S, Hoffman A. Structural investigation of xenon-ion-beam-irradiated glassy-carbon. Phys Rev B 1994;50(9):5905-17.

[38] Prawer S, Nugent KW, Lifshitz Y, Lempert GD, Grossman E, Kulik J, et al. Systematic variation of the Raman spectra of DLC films as a function of sp(2):sp(3) composition. Diam Relat Mater 1996;5(3-5):433-8.

[39] Fung AWP, Rao AM, Kuriyama K, Dresselhaus MS, Dresselhaus G, Endo M, et al. Raman-scattering and electrical-conductivity in highly disordered activated carbonfibers. J Mater Res 1993;8(3):489-500.

[40] Knight DS, White WB. Characterization of diamond films by Raman-spectroscopy. J Mater Res 1989;4(2):385-93.

[41] Papworth AJ, Kiely CJ, Burden AP, Silva SRP, Amaratunga GAJ. Electron-energy-loss spectroscopy characterization of the $\mathrm{sp}(2)$ bonding fraction within carbon thin films. Phys Rev B 2000;62(19):12628-31.

[42] Yan JY, Chen FR, Kai JJ. Mapping of sp(2)/sp(3) in DLC thin film by signal processed ESI series energy-loss image. J Electron Microsc 2002;51(6):391-400.

[43] Ponsonnet L, Donnet C, Varlot K, Martin JM, Grill A, Patel V. EELS analysis of hydrogenated diamond-like carbon films. Thin Solid Films 1998;319(1-2):97-100.

[44] Nolan PE, Schabel MJ, Lynch DC, Cutler AH. Hydrogen control of carbon deposit morphology. Carbon 1995;33(1):79-85.

[45] Xu GC, Chen BB, Shiki H, Katsumata T, Takikawa H, Sakakibara T, et al. Parametric study on growth of carbon nanocoil by catalytic chemical vapor deposition. Jpn J Appl Phys 2005;44(4A):1569-76.

[46] Shafarman WN, Koon DW, Castner TG. Dc conductivity of arsenic-doped silicon near the metal-insulator transition. Phys Rev B 1989;40(2):1216-31.

[47] Castner TG. Hopping conduction in the critical regime approaching the metal-insulator transition. In: Pollak M, Shklovshii BI, editors. Hopping transport in solids. Amsterdam: Elsevier Science;1991. p. 1-47.

[48] Chauvet O, Forro L, Bacsa W, Ugarte D, Doudin B, Deheer WA. Magnetic anisotropies of aligned carbon nanotubes. Phys Rev B 1995;52(10):R6963-6.

[49] Abrahams E, Anderson PW, Licciardello DC, Ramakrishnan TV. Scaling theory of localization: absence of quantum diffusion in two dimensions. Phys Rev Lett 1979;42(10):673-6. 